\title{
Relativistic Vlasov-Uehling-Uhlenbeck equation for nucleus-nucleus collisions
}

\author{
Q. Li, J. Q. Wu, and C. M. Ko \\ Cyclotron Institute and Center for Theoretical Physics, Texas A\&M University, College Station, Texas 77843
}

(Received 22 August 1988)

\begin{abstract}
A relativistic Vlasov-Uehling-Uhlenbeck equation for the nuclear phase-space distribution function is derived from the Walecka model through the use of semiclassical, local, and Born approximations.
\end{abstract}

The Vlasov-Uehling-Uhlenbeck (VUU) model introduced by Bertsch et al. ${ }^{1}$ for heavy-ion collisions has been used extensively in the study of the dynamics of heavyion collisions, ${ }^{2,3}$ particle productions from such reactions, ${ }^{4,5}$ and the properties of nuclear matter under extreme conditions. ${ }^{6,7}$ Although this model is carried out with the relativistic kinematics, it ignores the explicit exchange of mesons between nucleons, the small component of the nucleon wave function, and the negative energy states in the vacuum. As a first step towards a fully relativistic formulation of this model, we have recently constructed a relativistic VUU model for high-energy heavy-ion collisions. ${ }^{8-10}$ In this model, the mean field is obtained from the Walecka model ${ }^{11}$ in which the nucleon-nucleon interaction is described by the exchange of an attractive scalar meson and a repulsive vector meson. For the collision term, the nucleon-nucleon cross section in the free space is used as in the normal VUU model. We have successfully solved this model and applied it to heavy-ion collisions. Similar studies have been reported by Blättel et al. ${ }^{12}$ It is therefore of interest to derive consistently the collision term from the Walecka model as well. We shall pursue here such a derivation by generalizing the nonrelativistic approach of Refs. 13 and 14.

In the Walecka model, only the nucleon field $\psi$, the scalar-meson field $\phi$, and the vector-meson field $V^{\mu}$ are included. Other mesons such as the pion and the rho meson are neglected as they do not contribute to the nuclear binding energy in the mean-field approximation. However, they need to be included in the calculation of the nucleon-nucleon cross section. Since it is straightforward to include these other mesons, we neglect them in the following for simplicity. The Lagrangian density in the Walecka model is given by

$$
\begin{aligned}
L(x)= & \bar{\psi}\left[\gamma^{\mu}\left(i \partial_{\mu}-g_{v} V_{\mu}\right)-\left(m-g_{s} \phi\right)\right] \psi \\
& +\frac{1}{2}\left(\partial_{\mu} \phi \partial^{\mu} \phi-m_{s}^{2} \phi^{2}\right)-\frac{1}{4} F^{\mu v} F_{\mu v}+\frac{1}{2} m_{v}^{2} V^{\mu} V_{\mu},
\end{aligned}
$$

where

$$
F_{\mu \nu}=\partial_{\mu} V_{v}-\partial_{\nu} V_{\mu} .
$$

The masses $m, m_{s}$, and $m_{v}$ are for the nucleon, the scalar meson, and the vector meson, respectively; $g_{s}$ and $g_{v}$ are the coupling constants. The Green's function for the nucleon is defined as usual by

$$
i G\left(x_{1}, x_{2}\right)=\left\langle\left|T\left[\psi\left(x_{1}\right) \bar{\psi}\left(x_{2}\right)\right]\right|\right\rangle,
$$

where $T$ is the time ordering operator defined on a time contour as discussed in Ref. 14. Here, $\langle\cdots\rangle$ denotes the expectation value in the nuclear many-body state. The nucleon Green's function satisfies the following equation:

$$
\left(i \gamma_{\mu} \partial_{x_{1}}^{\mu}-m\right) i G\left(x_{1}, x_{1^{\prime}}\right)=i \delta\left(x_{1}-x_{1^{\prime}}\right)+\int d^{4} u \Sigma\left(x_{1}, u\right) i G\left(u, x_{1^{\prime}}\right),
$$

where $\Sigma$ is the nucleon self-energy.

Since the Walecka model is an effective field theory for nucleon-nucleon interaction in the nuclear matter, we shall consider only the first and second orders in calculating the nucleon self-energy, corresponding to the Hartree-Fock (HF) and Born terms, respectively. They are shown diagrammaticaly in Fig. 1, where the solid line denotes the nucleon while the dashed line represents either the scalar or the vector mesons. The first-order self-energy is real and can be written as ${ }^{15}$

$$
\Sigma_{\mathrm{HF}}(x)=\Sigma_{s}(x)+i \gamma_{\mu} \Sigma_{v}^{\mu}(x),
$$

and can be absorbed into the effective mass and momentum of the nucleon. Defining the nucleon effective mass by $m(x)=m+\Sigma_{s}(x)$, we can then rewrite Eq. (3) as

$$
\left\{i \gamma_{\mu}\left[\partial_{x_{1}}^{\mu}-\Sigma_{v}^{\mu}\left(x_{1}\right)\right]-m\left(x_{1}\right)\right\} i G\left(x_{1}, x_{1^{\prime}}\right)=i \delta\left(x_{1}-x_{1^{\prime}}\right)+\int d^{4} u \Sigma_{c}\left(x_{1}, u\right) i G\left(u, x_{1^{\prime}}\right)
$$

where $\Sigma_{c}$ is the second-order self-energy which, as shown later, leads to the collision term as in the nonrelativistic kinetic theory. 
As in Ref. 14, the nucleon Green's function $G\left(x_{1}, x_{1^{\prime}}\right)$ and self-energy $\Sigma_{c}\left(x_{1}, x_{1^{\prime}}\right)$ can be written as

$$
\begin{aligned}
& G\left(x_{1}, x_{1^{\prime}}\right)=\theta\left(t_{1}, t_{1^{\prime}}\right) G^{>}\left(x_{1}, x_{1^{\prime}}\right)+\theta\left(t_{1^{\prime}}, t_{1}\right) G^{<}\left(x_{1}, x_{1^{\prime}}\right), \\
& \Sigma_{c}\left(x_{1}, x_{1^{\prime}}\right)=\theta\left(t_{1}, t_{1^{\prime}}\right) \Sigma^{>}\left(x_{1}, x_{1^{\prime}}\right)+\theta\left(t_{1^{\prime}}, t_{1}\right) \Sigma^{<}\left(x_{1}, x_{1^{\prime}}\right),
\end{aligned}
$$

where $\theta\left(t_{1}, t_{1^{\prime}}\right)$ is 1 or 0 depending on whether $t_{1}$ is later or earlier than $t_{1^{\prime}}$ on the contour. Substituting Eqs. (6) and (7) into Eq. (5), we then find

$$
\begin{aligned}
\left\{\gamma_{\mu}\left[i \partial_{x_{1}}^{\mu}-\Sigma_{v}^{\mu}\left(x_{1}\right)\right]-m\left(x_{1}\right)\right\} i G^{<}\left(x_{1}, x_{1^{\prime}}\right)= & \int_{-\infty}^{t_{1}} d^{4} x_{2}\left[\Sigma^{>}\left(x_{1}, x_{2}\right)-\Sigma^{<}\left(x_{1}, x_{2}\right)\right] i G^{<}\left(x_{2}, x_{1^{\prime}}\right) \\
& -\int_{-\infty}^{t_{1}^{\prime}} d^{4} x_{2} \Sigma^{<}\left(x_{1}, x_{2}\right)\left[i G^{>}\left(x_{2}, x_{1^{\prime}}\right)-i G^{<}\left(x_{2}, x_{1^{\prime}}\right)\right],
\end{aligned}
$$

where the integration limits are for the time variable. There is no restriction to the spatial integrations.

Following Elze et al. ${ }^{16}$, we define $x=\left(x_{1}+x_{1^{\prime}}\right) / 2$ and $y=x_{1}-x_{1^{\prime}}$, and take the Fourier transform $\int d^{4} y e^{i p \cdot y}$ of the resulting equation. Making the semiclassical approximation, i.e., keeping only terms linear in $\hbar$, we obtain from Eq. (8)

$$
\left[\frac{i}{2} \gamma_{\mu} \partial_{x}^{\mu}+\gamma_{\mu} p^{* \mu}-m+\frac{i}{2}\left(\partial_{\mu}^{x} m\right) \partial_{p}^{\mu}-\frac{i}{2} \gamma_{\mu}\left(\partial_{v}^{x} p^{* \mu}\right) \partial_{p}^{v}\right] i G^{<}(x, p)=F_{c},
$$

where $p^{* \mu}=p^{\mu}-\Sigma_{v}^{\mu}$ is the kinetic momentum and $G^{<}(x, p)$ is the Fourier transform of the nucleon Green's function, i.e.,

$$
G^{<}(x, p)=\int d^{4} y e^{i p \cdot y} G^{<}(x+y / 2, x-y / 2) .
$$

The Fourier transform of the right-hand side of Eq. (8) is denoted by $F_{c}$. The real part of $F_{c}$ can also be absorbed into the effective mass and momentum of the nucleon and is neglected in the following. We then take the trace of both Eq. (9) and its product with $\gamma_{\mu}$ and equate the real and imaginary parts of the resulting equations. From the real part of the equations, the on-shell condition

$$
p^{* 2} \operatorname{Tr}\left[i G^{<}(x, p)\right]=m^{2} \operatorname{Tr}[i G<(x, p)],
$$

is obtained while the imaginary part leads to

$$
\frac{1}{2}\left\{\left[\partial_{x}^{\mu}-\left(\partial_{v}^{x} p^{* \mu}\right) \partial_{p}^{v}\right] \frac{p_{\mu}^{*}}{m}+\left(\partial_{x}^{\mu} m\right) \partial_{\mu}^{p}\right\} \operatorname{Tr}\left[i G^{<}(x, p)\right]=\operatorname{Im}\left(\operatorname{Tr} F_{c}\right),
$$

where Im denotes the imaginary part. In accordance with Eq. (11), we neglect the off-shell effect and set therefore the time limits of the two integrals in Eq. (8) equal. Furthermore, we assume, to be consistent with the semiclassical approximation, that the nucleon Green's function is smooth, so that its variation over a distance of $1 / p$ is neglected. Then we have explicitly,

$$
F_{c}=\int d^{4} y e^{i p \cdot y} \int_{-\infty}^{0} d^{4} y^{\prime}\left[\Sigma^{>}\left(y-y^{\prime}, x\right) i G^{<}\left(y^{\prime}, x\right)-\Sigma^{<}\left(y-y^{\prime}, x\right) i G^{>}\left(y^{\prime}, x\right)\right] .
$$

A similar equation can be obtained from the Hermitian conjugate of Eq. (8), i.e.,

$$
-\frac{1}{2}\left\{\left[\partial_{x}^{\mu}-\left(\partial_{v}^{x} p^{* \mu}\right) \partial_{p}^{v}\right] \frac{p_{\mu}^{*}}{m}+\left(\partial_{x}^{\mu} m\right) \partial_{\mu}^{p}\right\} \operatorname{Tr}\left[i G^{<}(x, p)\right]=\operatorname{Im}\left(\operatorname{Tr} F_{c}^{+}\right)
$$

where

$$
F_{c}^{+}=\int d^{4} y e^{i p \cdot y} \int_{0}^{\infty} d^{4} y^{\prime}\left[\Sigma^{<}\left(y-y^{\prime}, x\right) i G^{>}\left(y^{\prime}, x\right)-\Sigma^{>}\left(y-y^{\prime}, x\right) i G^{<}\left(y^{\prime}, x\right)\right] .
$$

Substracting Eq. (14) from Eq. (12), we arrive at the following equation:

$$
\left\{\left[\partial_{x}^{\mu}-\left(\partial_{v}^{x} p^{* \mu}\right) \partial_{p}^{v}\right] \frac{p_{\mu}^{*}}{m}+\left(\partial_{x}^{\mu} m\right) \partial_{\mu}^{p}\right\} \operatorname{Tr}\left[i G^{<}(x, p)\right]=\operatorname{Tr}\left[\Sigma^{>}(x, p) G^{<}(x, p)-\Sigma^{<}(x, p) G^{>}(x, p)\right] .
$$

Expressing the nucleon Green's function in terms of the kinetic momentum, i.e., $G^{<}\left(x, p^{*}\right)$, and making use of the equality

$$
\left.\partial_{x}^{\mu}\right|_{p=\text { const }}=\left.\partial_{x}^{\mu}\right|_{p^{*}=\text { const }}-\left(\partial_{x}^{\mu} \Sigma_{v v}\right) \partial_{p}^{v},
$$

where $\partial_{p}$ is the partial derivative with respect to the kinetic momentum $p^{*}$, and $\left.\partial_{x}\right|_{p, p}{ }^{*}=\operatorname{const}$ is evaluated with $p$ or $p^{*}$ being kept constant, we rewrite Eq. (16) as 


$$
\left\{\left[\partial_{x}^{\mu}-\left(\partial_{x}^{\mu} \Sigma_{v}^{v}-\partial_{x}^{v} \Sigma_{v}^{\mu}\right) \partial_{v}^{p^{*}}\right] \frac{p_{\mu}^{*}}{m}+\left(\partial_{x}^{\mu} m\right) \partial_{\mu}^{p^{*}}\right\} \operatorname{Tr}\left[i G^{<}\left(x, p^{*}\right)\right]=\operatorname{Tr}\left[\Sigma^{>}\left(x, p^{*}\right) G^{<}\left(x, p^{*}\right)-\Sigma^{<}\left(x, p^{*}\right) G^{>}\left(x, p^{*}\right)\right]
$$

To simplify the notation, we shall drop the vector meson in the derivation of the collision term and consider the scalar meson only. In the end of the derivation, it is straightforward to put back the contribution from the vector meson. In terms of the Fourier transform $\Delta(x, p)$ of the scalar meson propagator $\Delta\left(x_{1}, x_{2}\right)$ defined by

$$
i \Delta\left(x_{1}, x_{2}\right)=\left\langle\left|T\left[\phi\left(x_{1}\right) \phi^{+}\left(x_{2}\right)\right]\right|\right\rangle,
$$

the second-order nucleon self-energy can be calculated easily from the Born diagrams in Fig. 1. It is given by

$$
\begin{aligned}
\Sigma^{<}\left(x, p^{*}\right)=\int \frac{d^{4} p_{2}^{*}}{(2 \pi)^{4}} \int \frac{d^{4} p_{3}^{*}}{(2 \pi)^{4}} \int \frac{d^{4} p_{4}^{*}}{(2 \pi)^{4}} g_{s}^{4}(2 \pi)^{4} \delta^{4}\left(p^{*}+p_{2}^{*}-p_{3}^{*}-p_{4}^{*}\right) \\
\\
\times\left\{\Delta^{2}\left(x, p^{*}-p_{3}^{*}\right) G^{>}\left(x, p_{3}^{*}\right) \operatorname{Tr}\left[G^{>}\left(x, p_{2}^{*}\right) G^{<}\left(x, p_{4}^{*}\right)\right]\right. \\
\left.\quad-\Delta\left(x, p^{*}-p_{3}^{*}\right) \Delta\left(x, p^{*}-p_{4}^{*}\right) G^{<}\left(x, p_{3}^{*}\right) G^{>}\left(x, p_{2}^{*}\right) G^{>}\left(x, p_{4}^{*}\right)\right\},
\end{aligned}
$$

where the first term is the direct term while the second term is the exchange term. Interchanging the superscript " $>$ " with " $<$ " in Eq. (19) gives $-\Sigma^{<}\left(x, p^{*}\right)$.

To proceed further, we introduce the local approximation at the space-time point $x$, i.e., the field operator $\psi(x)$ is assumed to have the following expansion:

$$
\psi(x)=\sum_{p^{*}, s} U_{x}^{s}\left(p^{*}\right) e^{i p^{*} \cdot x} a_{x}\left(\mathbf{p}^{*}\right),
$$

where $U_{x}^{s}\left(p^{*}\right)$ is the spinor wave function for spin state $s$ and is normalized according to

$$
\operatorname{Tr} \sum_{s} U_{x}^{s}\left(p^{*}\right) \bar{U}_{x}^{s}\left(p^{*}\right)=4 m / p_{0}^{*}
$$

with 4 the spin-isospin degeneracy of the nucleon, and

$$
p_{0}^{*}=\omega \equiv\left(\mathbf{p}^{* 2}+m^{2}\right)^{1 / 2}
$$

because of the on-shell condition Eq. (11). The nucleon annihilation operator is denoted by $a_{x}\left(\mathrm{p}^{*}\right)$. The antinucleon part has been dropped in the present study. From Eqs. (2), (6), and (21) we have

$$
i G_{\alpha \beta}^{<}\left(x, p^{*}\right)=-\sum_{s} U_{x \alpha}^{s}\left(p^{*}\right) \bar{U}_{x \beta}^{s}\left(p^{*}\right) f\left(x, \mathrm{p}^{*}\right) 2 \pi \delta\left(p_{0}^{*}-\omega\right)
$$

and

$$
i G_{\alpha \beta}^{>}\left(x, p^{*}\right)=\sum_{s} U_{x \alpha}^{s}\left(p^{*}\right) \bar{U}_{x \beta}^{s}\left(p^{*}\right)\left[1-f\left(x, \mathbf{p}^{*}\right)\right] 2 \pi \delta\left(p_{0}^{*}-\omega\right) .
$$

The function $f\left(x, \mathrm{p}^{*}\right)$ is the seven-dimensional on-shell nucleon's phase-space distribution function defined by

$$
f\left(x, \mathrm{p}^{*}\right)=\left\langle\left|a_{x}^{+}\left(\mathrm{p}^{*}\right) a_{x}\left(\mathrm{p}^{*}\right)\right|\right\rangle .
$$

From Eq. (22), we can write

$$
\operatorname{Tr}\left[i G^{<}\left(x, p^{*}\right)\right]=-16 \pi \delta\left(p^{* 2}-m^{2}\right) m f\left(x, \mathbf{p}^{*}\right) .
$$

We then substitute it into the left-hand side of Eq. (18) and neglect the off-shell contributions by taking $\delta\left(p^{* 2}-m^{2}\right)$ to the left of the differential operators. For the right-hand side of Eq. (18), we use Eqs. (22) and (23). After some straightforward algebra, we obtain finally the relativistic VUU equation,

$$
\begin{aligned}
\frac{1}{p_{0}^{*}}\left\{\left[\partial_{x}^{v}-\left(\partial_{x}^{v} \Sigma_{v}^{v}-\partial_{x}^{v} \Sigma_{v}^{\mu}\right) \partial_{v}^{p^{*}}\right] p_{\mu}^{*}+m\left(\partial_{x}^{\mu} m\right) \partial_{\mu}^{p^{*}}\right\} f\left(x, \mathbf{p}^{*}\right) \\
=\int \frac{d \mathbf{p}_{2}^{*}}{(2 \pi)^{3}} \int \frac{d \mathbf{p}_{3}^{*}}{(2 \pi)^{3}} \int \frac{d \mathbf{p}_{4}^{*}}{(2 \pi)^{3}} \overline{|M|^{2}}(2 \pi)^{4} \delta^{3}\left(\mathbf{p}^{*}+\mathbf{p}_{2}^{*}-\mathbf{p}_{3}^{*}-\mathbf{p}_{4}^{*}\right) \delta\left(\omega+\omega_{2}-\omega_{3}-\omega_{4}\right) \\
\quad \times\left\{f\left(x, \mathbf{p}_{3}^{*}\right) f\left(x, \mathbf{p}_{4}^{*}\right)\left[1-f\left(x, \mathbf{p}^{*}\right)\right]\left[1-f\left(x, \mathbf{p}_{2}^{*}\right)\right]\right. \\
\left.\quad-f\left(x, \mathbf{p}^{*}\right) f\left(x, \mathbf{p}_{2}^{*}\right)\left[1-f\left(x, \mathbf{p}_{3}^{*}\right)\right]\left[1-f\left(x, \mathbf{p}_{4}^{*}\right)\right]\right\}
\end{aligned}
$$

where $\overline{|M|^{2}}$ is the spin and isospin average of the square of the nucleon-nucleon invariant scattering amplitude $M$ in the Born approximation, 


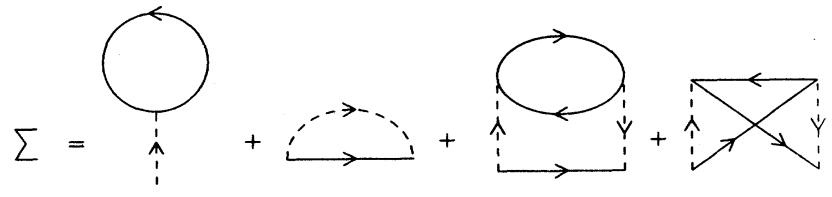

FIG. 1. First- and second-order nucleon self-energy. The solid curve denotes the nucleon while the dashed line represents either the scalar or the vector mesons.

$$
\begin{aligned}
M= & i g_{s}^{2}\left[\bar{U}_{x}^{s_{3}}\left(p_{3}^{*}\right) U_{x}^{s_{1}}\left(p_{1}^{*}\right) \Delta\left(x, p_{1}^{*}-p_{3}^{*}\right) \bar{U}_{x}^{s_{4}}\left(p_{4}^{*}\right) U_{x}^{s_{2}}\left(p_{2}^{*}\right)\right. \\
& \left.-\bar{U}_{x}^{s_{4}}\left(p_{4}^{*}\right) U_{x}^{s_{1}}\left(p_{1}^{*}\right) \Delta\left(x, p_{1}^{*}-p_{4}^{*}\right) \bar{U}_{x}^{s_{3}}\left(p_{3}^{*}\right) U_{x}^{s_{2}}\left(p_{2}^{*}\right)\right]
\end{aligned}
$$

The vector meson can be straightforwardly included by using the scattering amplitude calculated in the Born approximation with both scalar and vector mesons. Other mesons such as the pion and $\rho$ meson can also be taken into account in a similar way.

The mean-field part of the relativistic VUU equation has the same form as that derived in Refs. 8 and 16. The collision term also has a similar form as that used in our previous calculations in that it contains both the Pauliblocking factors and the nucleon-nucleon cross section. ${ }^{9}$ According to the preceding derivation, the nucleonnucleon cross section in the Born approximation can be calculated with the exchange of both the scalar and the vector mesons, and possibly also the isovector mesons such as the pion and the rho meson. Once the nucleonnucleon cross section in the free space can be fitted in an average way by such an effective meson model, then the density dependence of the nucleon-nucleon cross section in the nuclear matter can be studied by including the self-energy of the mesons. Such studies have already been explored by Bertsch et al. ${ }^{17}$ for the nucleon-nucleon inelastic cross section and by $\mathrm{Wu}$ et al. ${ }^{18}$ for the kaon production cross section.

One of the authors (Ko) thanks Dr. Mitsuru Tohyama for a number of useful conversations. He also appreciates Professor Ulrich Mosel for the warm hospitality during his recent visit at the University of Giessen where part of this work was carried out. This work was supported in part by the National Science Foundation under Grant No. PHY -8608149 and the Robert A. Welch Foundation under Grant No. A-1110.
${ }^{1}$ G. F. Bertsch, H. Kruse, and S. Das Gupta, Phys. Rev. C 29, 673 (1984).

2J. J. Molitoris and H. Stöcker, Phys. Rev. C 32, 346 (1985).

${ }^{3}$ J. Aichelin and G. F. Bertsch, Phys. Rev. C 31, 1730 (1985).

${ }^{4}$ W. Bauer et al., Phys. Rev. C 34, 2127 (1986).

${ }^{5}$ C. M. Ko and J. Aichelin, Phys. Rev. C 35, 1976 (1987).

${ }^{6}$ H. Kruse, B. V. Jacak, and H. Stöcker, Phys. Rev. Lett. 52, 289 (1985).

${ }^{7}$ J. Aichelin and C. M. Ko, Phys. Rev. Lett. 55, 2661 (1985).

${ }^{8}$ C. M. Ko, Q. Li, and R. Wang, Phys. Rev. Lett. 59, 1084 (1987).

${ }^{9}$ C. M. Ko and Q. Li, Phys. Rev. C 37, 2270 (1988).
${ }^{10}$ Q. Li and C. M. Ko, Mod. Phys. Lett. A 3, 465 (1988).

${ }^{11}$ J. D. Walecka, Ann. Phys. (N.Y.) 83, 491 (1974).

${ }^{12}$ B. Blättel, V. Koch, W. Cassing, and U. Mosel, Phys. Rev. C 38, 1767 (1988).

${ }^{13}$ L. P. Kadanoff and G. Baym, Quantum Statistical Mechanics (Benjamin, New York, 1962).

${ }^{14}$ P. Danielewicz, Ann. Phys. (N.Y.) 152, 239 (1984).

${ }^{15}$ S. A. Chin, Ann. Phys. (N.Y.) 108, 301 (1977).

${ }^{16}$ H. T. Elze et al., Mod. Phys. Lett. A 2, 451 (1987).

${ }^{17}$ G. F. Bertsch, G. E. Brown, V. Koch, and B. A. Li, Nucl. Phys. A490, 745 (1989).

${ }^{18}$ J. Q. Wu and C. M. Ko, Texas A\&M University report, 1988. 